\title{
Ambiguity Function Analysis for Passive Radar System Performance
}

\author{
Nicholas Johnson, Mohamed Chergui, Oren Sternberg, John D. Rockway, \\ and W. Linwood Jones ${ }^{1}$, Life Fellow, IEEE \\ 1. Central Florida Remote Sensing Laboratory, ECE Dept, University of Central Florida
}

\begin{abstract}
Passive coherent location (PCL) systems can employ a variety of terrestrial broadcast signals for target detection and ranging. Through analysis of the ambiguity function, one can determine a signal's suitability for this application. The main parameters of concern are amplitude, range resolution, Doppler resolution and sidelobe ratios. This paper presents an analysis of several recorded signals in terms of their suitability for PCL. We also discuss the effects of compromise when choosing between performance in range or Doppler resolution, signal processing gain, and surveillance volume.
\end{abstract}

Keywords-Passive Coherent Location; Passive Radar; Bistatic Radar; Radar Signal Processing; Ambiguity Function

\section{INTRODUCTION}

A passive coherent location (PCL) system uses non-cooperative signals of opportunity as bistatic illuminators to detect targets of interest [1]. These signals have typically been broadcast FM radio and HDTV, although several papers have been published attempting to use signals such as GPS and cell phone networks $[15,16,17]$. Using these signals to perform traditional radar signal processing functions can be challenging. One of the biggest difficulties is eliminating enough of the direct path signal from the surveillance channel such that a reasonable target can be detected [2]. This can be especially difficult when a target is suspected near the same azimuth as the transmitter of opportunity. Another challenge of PCL implementation is the tradeoff between signal processing gain and range resolution [13]. It is possible to magnify a target in the presence of clutter and noise by increasing the coherent processing interval (CPI). However when using a continuous wave $(\mathrm{CW})$ signal as the illuminator, lengthening the CPI can cause a missed detection in the appropriate range bin $[4,5]$. Additionally, range resolution is determined by the sampling rate of incoming IQ data. If the PCL system has little processing power to accommodate large sampling rates, compromises will have to be made.

In this paper we present several recorded signals including HF CODAR, FM radio, and HDTV and their corresponding ambiguity functions. We will individually analyze each one to illustrate the effects each signal has on target detection capabilities. Features to be examined include range, Doppler resolution, peak side-lobe level ratio (PLSR) and integrated sidelobe ratio (ISLR). Lastly, we demonstrate a target response within an implemented PCL system.

\section{DAta Collection Methods}

The scenario geometry for the proposed PCL system is depicted in Fig. 1. The collection site is located on the cliffs of Point Loma peninsula, located in San Diego. The sets of transmitted signals and their locations are also depicted in the figure. The first set of signals to be captured are from two local, high-frequency (HF) coastal ocean dynamics applications radars (CODAR) controlled by Scripps Institute of Oceanography. These signals were recorded at 5.3 (SDSL) and $24 \mathrm{MHz}$ (SDPL). The second signals came from the FM Radio Stations 98.8 and $101.5 \mathrm{MHz}$, which are located 25 miles southeast of the collection site. The Effective Radiated Power for both is $50 \mathrm{~kW}$ and each has an omni-directional pattern. The broadcast TV stations at 497 $\mathrm{MHz}$ and $500 \mathrm{MHz}$ are located 18 miles directly east of the collection site which has a broadcast ERP of 355 and $328 \mathrm{~kW}$, respectively. At the collection site, two directional antennas were utilized. One antenna was pointed toward $135^{\circ}$ (SE) and the other toward $80^{\circ}$ (ENE). Collections were performed in clear weather conditions during the daytime.

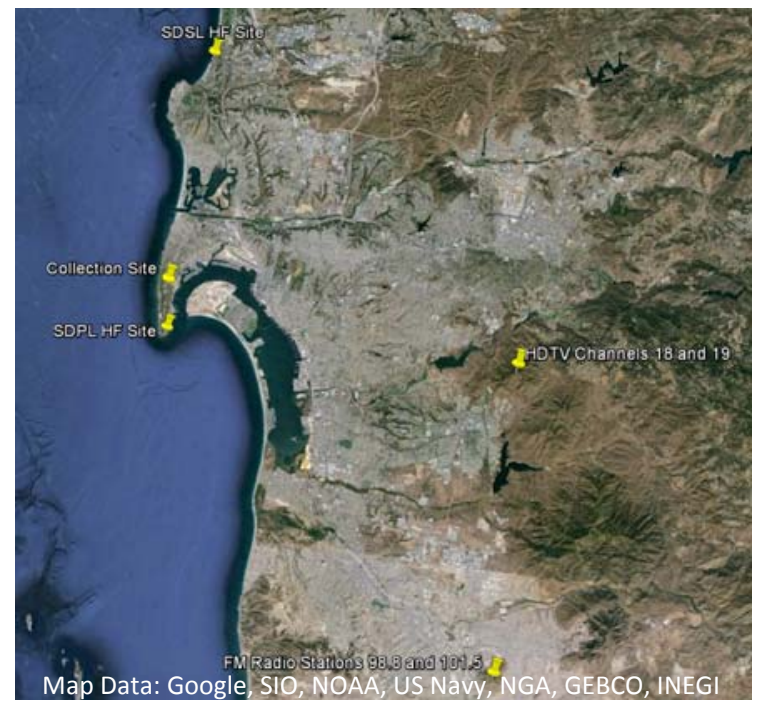

Figure 1. PCL Geometry for FM/HDTV Signal Collection

The data for this paper was collected using two software defined radios (SDR) and a pair of matched log-periodic antennas. The SDRs were GPS synchronized during RF data collection, see Figure 2 for schematic. 
For the signal collections, we utilized two Channel Master CM3016 log periodic antennas with a $75 \mathrm{Ohm}$ balun (300-75 ohms), matched to 50 Ohms (Pasternak). The matched Antennas were then connected to an SDR Ettus URSP N210 with a Basic RX daughterboard and GPS. The SDRs were connected via gigabit Ethernet to a Linux PC (Ubuntu 14.04) and GPS antenna. A python/C++ script acted as a trigger synchronizing the GPS and the PC clock. The triggered collections were stored on the PC for post processing, See Table 1 for Hardware and software specifications.

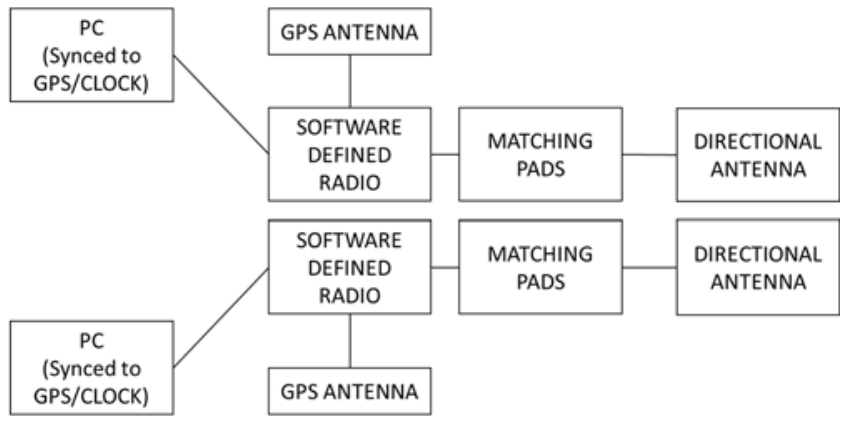

Figure 2. Experimental PCL Collection Setup

The selected sample rate for the synchronous FM collections were 2.4 mega samples per second at FM frequencies of 101.1 $\mathrm{MHz}$ and Bandwidth of $2.4 \mathrm{MHz}$, the durations of collections ranged from 20-30 seconds.

TABLE I. EXPERIMENTAL PCL HARDWARE AND SOFTWARE

\begin{tabular}{|c|c|}
\hline \multicolumn{2}{|c|}{ HARDWARE } \\
\hline Device & Model \\
\hline Software Defined Radio (2) & USRP N210/BasicRx/GPS \\
\hline Antenna (2) & Channel Master, CM3016 \\
\hline PC (2) - Lu & Intel NUC i7 \\
\hline Matching Pads (2) & 75-50 Pasternak \\
\hline GPS Antennas (2) & GPS-00464 (Sparkfun) \\
\hline Cables (2) & RG-9 Coax Cable \\
\hline \multicolumn{2}{|c|}{ SOFTWARE } \\
\hline GNU RADIO, C, Python, OCTAVE, MATLAB \\
OS: Ubuntu 14.04
\end{tabular}

Once the data was collected for all signals of interest, analysis was performed with post-processing methods including calculation and analysis of each signal's ambiguity function.

\section{Signal ANALYsis USING The AmBIGUITy FunCtion}

The ambiguity function is used in traditional radar waveform applications as a measure of capabilities in terms of object detection, rejection of clutter, range and Doppler resolution, accuracy of measurements, and overall ambiguity $[1,12]$. However in passive radar systems, several of these features can be determined in near real-time through calculation of a signal's ambiguity function. To increase the chances of detection and discrimination of closely-spaced targets, a radar waveform designer defines qualitative traits as range resolution, peak-tosidelobe ratio, and integrated sidelobe ratio [13].
The calculation of an ambiguity function is performed by passing a waveform through its own matched filter. In the case of passive radar, we define this as autocorrelation because we correlate a signal of interest with itself. For the monostatic case, the ambiguity function is defined in [15] as

$$
\left|X(\tau, f)^{2}\right|=\left|\int_{-\infty}^{\infty} s_{t}(t) s_{t}^{*}(t-\tau) e^{j 2 \pi f t} d t\right|^{2}
$$

where $X(\tau, f)$ is the ambiguity function dependent on time delay $(\tau)$ and Doppler frequency $(f)$, and $\mathrm{s}(t)$ represents a complex baseband signal.

In [6], Tsao formulates a different equation for the bistatic case, making the argument that time delay and Doppler frequency are not linear functions of range and velocity because of geometric considerations. His proposed ambiguity function is

$$
\begin{aligned}
\mid X\left(R_{R H},\right. & R_{R a}, V_{a}, \theta_{R},\left.L\right|^{2} \\
& =\mid \int \tilde{f}\left(t-\tau_{a}\left(R_{R a}, \theta_{R}, L\right)\right) \tilde{f}^{\prime}((t \\
& \left.-\tau_{a}\left(R_{R a}, \theta_{R}, L\right)\right) \exp \left[-j\left(\omega_{D H}\left(R_{R H}, V_{H}, \theta_{R}, L\right)\right.\right. \\
& \left.\left.-\omega_{D A}\left(R_{R a}, V_{a}, \theta_{R}, L\right)\right) t\right]\left.d t\right|^{2}
\end{aligned}
$$

which incorporates bistatic range, angles, and radial velocities from the positions of both the transmitter and receiver. Tsao also shows through simulation that the bistatic ambiguity function shape is dependent on the bistatic geometry, meaning that range and Doppler resolutions will also vary. Taking into account bistatic geometry is important for characterizing situations when tracking or searching for a target, or when using multiple geographically separated transmitters of opportunity. For this paper we rely on the monostatic ambiguity function calculation due to the fact that we are not performing target detection, only analyzing a signal based on its autocorrelation function.

The most obvious characteristic when searching for useful signals is amplitude. The amplitude of the calculated ambiguity function can be increased by lengthening the coherent processing interval (CPI). It can be seen in Figure 3 below that doubling the number of samples used in the autocorrelation increases the amplitude by $3 \mathrm{~dB}$. The disadvantage of increasing the CPI is the chance that a fast moving target will migrate through range bins quicker than a detection can be made. 


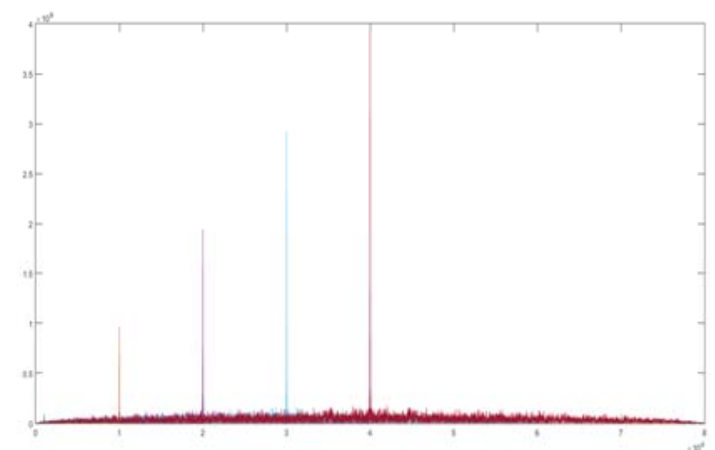

Figure 3. Comparison of Increasing Ambiguity Function Amplitude for $1000,10000,20000,30000$, and 40000 samples

Another feature that must be studied is the leakage of direct path signal into the surveillance channel. For this reason we can use an adaptive filter to cancel the unwanted signal. However at some azimuths within $90^{\circ}$ of the transmit location, it may be difficult to remove the direct path signal without physical isolation between receive antennas.

Another feature that can be derived from the ambiguity function is range resolution. The range resolution $\tau$ for a given signal of opportunity is determined by the relationship given in (3):

$$
\Delta R=\frac{c}{2 B}
$$

where $c$ equals the speed of light and $B$ is the signal bandwidth. For example, the majority of FM radio channels are allocated $200 \mathrm{kHz}$ of bandwidth. This corresponds to a relative range resolution of 750 meters. However in reality most FM channels do not use all $200 \mathrm{kHz}$ of bandwidth constantly, so the range resolution will vary in time with respect to modulation of the channel. Results presented in [9] illustrate this concept.

The Doppler resolution in a PCL system is largely dependent on the frequency of the signal and is given by the relationship

$$
\Delta f=\frac{2 v}{c} * f_{c}
$$

However for the purposes of this paper, a target velocity does not exist, so we make $\Delta f=1$ and solve for the minimum target velocity. The Doppler resolution in also dependent on the modulation content of the signal.

The sidelobe levels of the ambiguity function will indicate a signals ability to resolve a target response in both the range and Doppler dimensions. Two metrics we will use to characterize a signal's abilities are peak to sidelobe ratio (PSLR) and integrated sidelobe ratio (ISLR). PSLR shows a signals ability to resolve targets with various amplitude responses in the same range bin. ISLR indicates a signal's ability to resolve multiple targets in the same range cell. The equations for PSLR and ISLR are given below [11]:

$$
\begin{gathered}
P S L R=\frac{1}{A_{0}^{2}} \max \left\{A_{n}^{2}\right\}, n \neq 0 \\
I S L R=\frac{1}{A_{0}^{2}} \sum_{n=1}^{N}\left\{A_{n}^{2}\right\}
\end{gathered}
$$

\section{ANALYSIS OF COLLECTED Signals}

The first set of analysis we present are from the local HF CODAR. The calculated ambiguity function for these signals are presented in Figures 4 and 5. The 5.3 MHz CODAR signal has a bandwidth of $25 \mathrm{kHz}$, compared to the $17.6 \mathrm{MHz}$ signal bandwidth of $75 \mathrm{kHz}$. This means that the $17.6 \mathrm{MHz}$ CODAR will have a better range resolution $(2 \mathrm{~km})$ compared to the 5.3 $\mathrm{MHz}$ signal $(6 \mathrm{~km})$. Also, by using the HF signals, we are able to resolve target velocities between approximately 6 and $28 \mathrm{~m} / \mathrm{s}$.

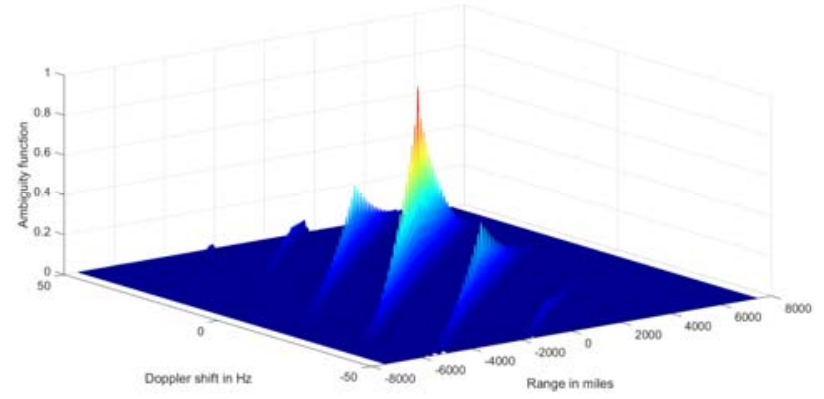

Figure 4. Calculated Ambiguity Function for HF CODAR Signal 5.3MHz.

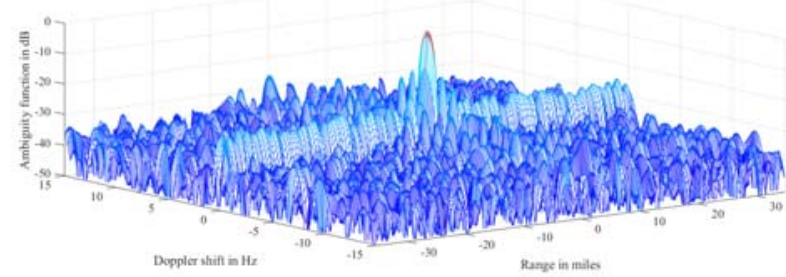

Figure 5. Calculated Ambiguity Function for HF CODAR Signal 17.6MHz.

The next set of signals collected were broadcast FM radio signals. The ambiguity functions for two FM radio signals can be seen in Figures 6 and 7. Both of these channels are allocated $200 \mathrm{kHz}$ bandwidth, however the most we observed was roughly $100 \mathrm{kHz}$. This corresponds to a range resolution span of 750$1500 \mathrm{~m}$. 


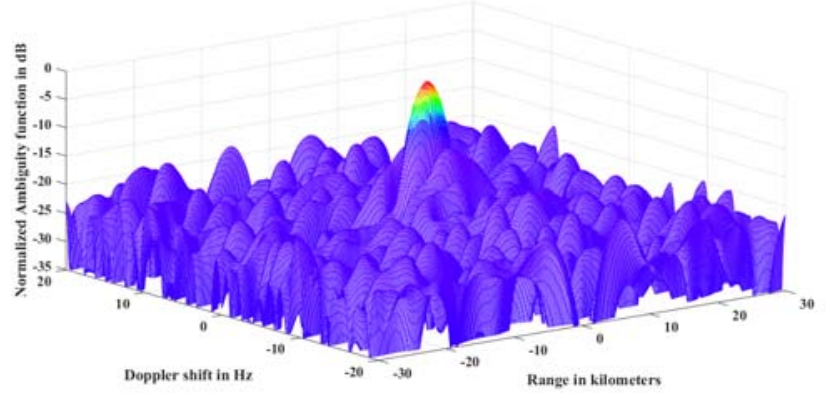

Figure 6. Calculated Ambiguity Function for FM Radio Station 98.8 MHz.

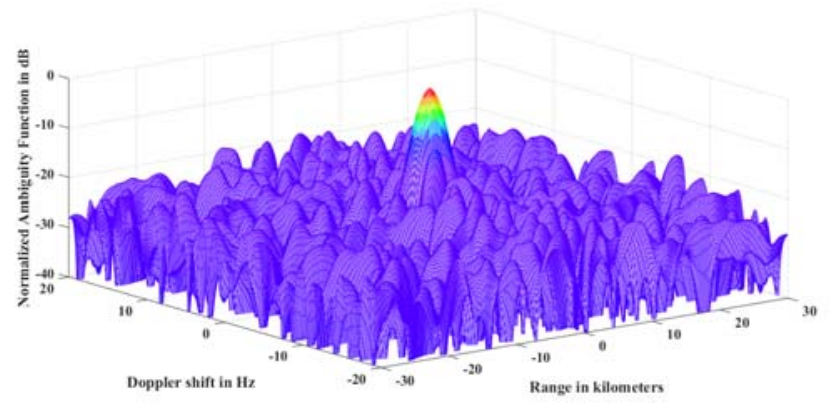

Figure 7. Calculated Ambiguity Function for FM Radio Station 101.5 MHz.

The last set of collected signals were broadcast HDTV channels. The ambiguity functions can be seen in Figures 8 and 9. The allocated bandwidth of an HDTV channel is $6 \mathrm{MHz}$. This corresponds to a best possible range resolution of $25 \mathrm{~m}$.

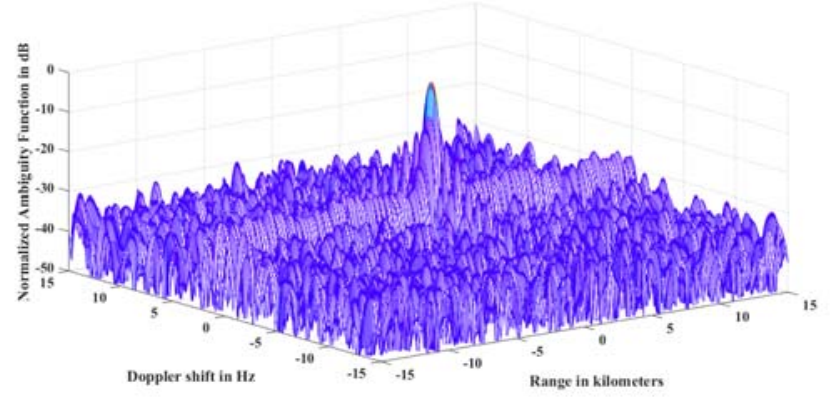

Figure 8. Calculated Ambiguity Function for HDTV Station $494 \mathrm{MHz}$.

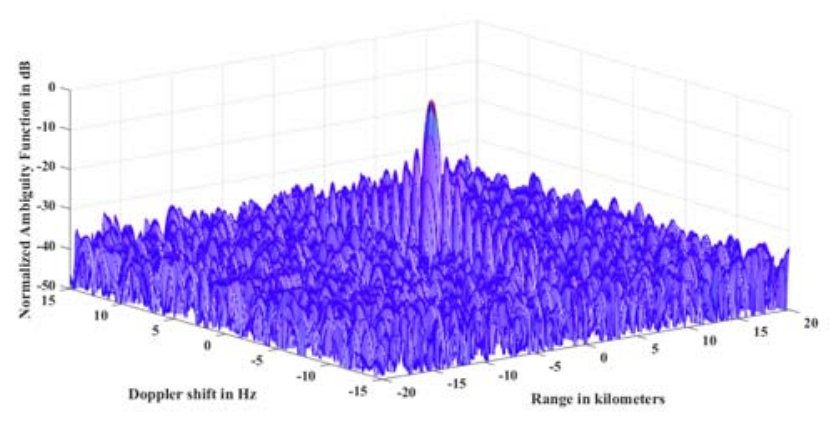

Figure 9. Calculated Ambiguity Function for HDTV Station $500 \mathrm{MHz}$.
Table II summarizes the characteristics of each collected signal. It can be seen that the main driver for range and Doppler resolution for this signal set is frequency.
TABLE II. COMPARISON BetWeEn COLLECTED Signals

\begin{tabular}{|l|c|c|c|c|c|c|}
\hline Features & $\begin{array}{c}\mathbf{5 . 3} \\
\text { (HF) }\end{array}$ & $\begin{array}{c}\mathbf{1 7 . 6} \\
\text { (HF) }\end{array}$ & $\begin{array}{c}\mathbf{9 8 . 8} \\
\text { (FM) }\end{array}$ & $\begin{array}{c}\mathbf{1 0 1 . 5} \\
\text { (FM) }\end{array}$ & $\begin{array}{c}\text { 495 } \\
\text { (HDTV) }\end{array}$ & $\begin{array}{c}\mathbf{5 0 0} \\
\text { (HDTV) }\end{array}$ \\
\hline $\begin{array}{l}\text { Range } \\
\text { Resolution } \\
\text { (m) }\end{array}$ & 6000 & 2000 & 750 & 750 & 25 & 25 \\
\hline $\begin{array}{l}\text { Velocity } \\
\text { Resolution } \\
\text { (m/s) }\end{array}$ & 28.3 & 5.88 & 1.52 & 1.48 & 0.30 & 0.3 \\
\hline $\begin{array}{l}\text { PSLR } \\
\text { (range } \\
\text { dimension) }\end{array}$ & .97 & .95 & 1.25 & 1.23 & 1.17 & 1.19 \\
\hline $\begin{array}{l}\text { ISLR } \\
\text { (range } \\
\text { dimension) }\end{array}$ & 0.9 & 0.89 & 1.43 & 1.45 & 1.52 & 1.53 \\
\hline
\end{tabular}

As a final measure of analysis we discuss the suppression of the direct path signal from that of the reflected target. Due to the antenna positions for this experiment, the direct signal was collected at a $90^{\circ}$ azimuth from the surveillance signal. This resulted in a large amount of unwanted direct signal leaking into the surveillance channel. In an effort to reduce the undesired signal we use a least mean squares (LMS) filter approach. This method treats the direct path signal as interferer and the surveillance signal as signal plus interferer. Using this method we are able to realize approximately $20 \mathrm{~dB}$ of suppression.

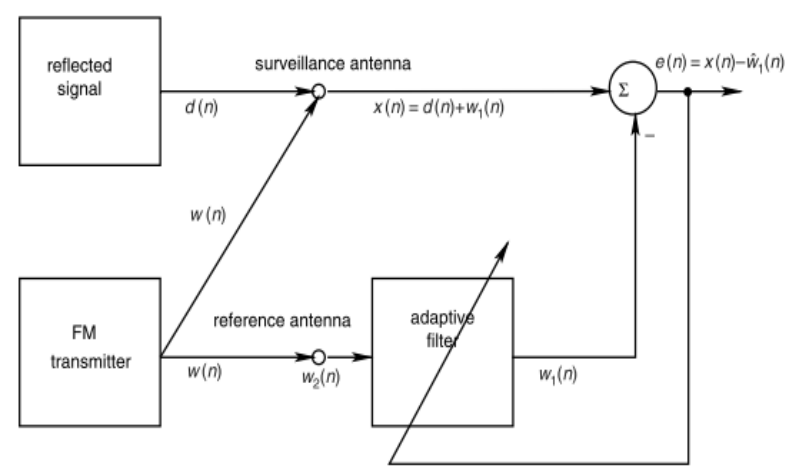

Figure 10. LMS Filter Block Doagram 
In Figure 11 we illustrate the range/Doppler response of a target that was detected using FM radio station 101.5. The target is a commercial helicopter that appears to be approximately $9 \mathrm{~km}$ from the collection site moving roughly $14 \mathrm{~m} / \mathrm{s}(33 \mathrm{mph})$. The target was verified using an ADS-B receiver. The strong target response can be seen despite the clutter. One can also see the large amount of clutter due to the terrain approximately $35 \mathrm{~km}$ east of San Diego.

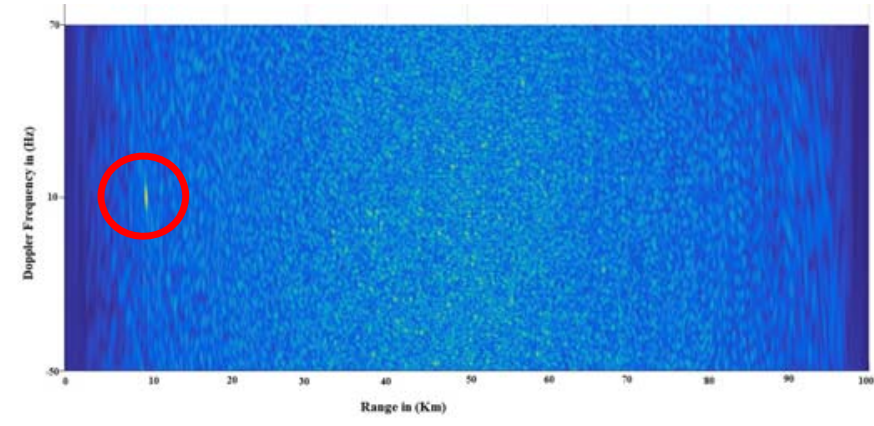

Figure 11. Target Detection using Range-Doppler Reponse of FM 101.5 MHz Radio Signal.

\section{CONCLUSION AND FUTURE WORK}

This work presents analysis of several signals from the perspective of passive radar applications. Using the ambiguity function calculated from each signal, we illustrated that amplitude, range resolution, Doppler resolution, and sidelobe levels are all important factors when choosing a signal for passive radar functions. In addition, we show that when choosing signals of opportunity the electromagnetic environment is another important factor. The direct signal from the transmitter of opportunity can overwhelm the target detection signal if it cannot be suppressed with either signal processing cancellation techniques or spatial separation. Future work will include continued collection of signals of interest with targets present. This will allow us to explore the bistatic ambiguity functions presented in Tsao [7] and Baker [14].

\section{REFERENCES}

[1] Griffiths, H.D., "From a Different Perspective: Principles, Practice, and Potential of Bistatic Radar", 2003 Proceedings of the International Conference on Radar.

[2] Griffiths, H., Baker, C., "Measurement and Analysis of Ambiguity Functions of Passive Radar Transmissions", International Radar Conference, 2005.

[3] M. Skolnik, Radar Handbook, 3rd ed, New York: McGraw Hill, 2008, pp.23.1-23.31.

[4] Willis, Nicholas J., Griffiths, Hugh D., Advances in Bistatic Radar, Edison, NJ: SciTech Publishing, 2007.

[5] Cherniakov, Mikhail, Bistatic Radar Principles and Practice, West Sussex, England: John Wiley and Sons, Ltd, 2007.

[6] Tsao, T., Slamani, M., Varshney, P., Weiner, D., Schwarzlander, H., "Ambiguity Function for a Bistatic Radar", IEEE Transactions on Aerospace and Electronic Systems, Vol. 33, No. 3, pp. 1041-1051, July 1997.

[7] Tasdelen, A., Koymen, H., "Range Resolution Improvement in Passive Coherent Location Radar Systems Using Multiple FM Radio Channels", IET Forum on Waveform Diversity and Design in Communications, Radar, and Sonar, 2006.

[8] Szlachetko, B., Lewandowski, A., "A Multichannel Receiver of the Experimental FM Based Passive Radar Using Software Defined Radio Technology", International Journal of Electronics and Telecommunications, 2012, Vol. 58, No. 4, pp. 301-306.

[9] Griffiths, H.D., Baker, C.J., Ghaleb, H., Ramakrishnan, R., Willman, E., "Measurement and Analysis of Ambiguity Functions of Off-Air Signals for Passive Coherent Location", IEEE Electronics Letters, June 2003, Vol. 39, No. 13.

[10] Richards, Mark A., Fundamentals of Radar Signal Processing, $2^{\text {nd }}$ ed., McGraw Hill Education, 2014

[11] A. Lauri, F. Colone, R. Cardinali, C. Bongioanni, P. Lombardo, "Analysis and Emulation of FM Radio Signals for Passive Radar", IEEE Aerospace Conference 2007

[12] Melvin, William L., Scheer, James A., Principles of Modern Radar Advanced Techniques, Edison, NJ: SciTech Publishing, 2007

[13] Baker, C.J., Griffiths, H.D., Papoytsis, I., "Passive Coherent Location Radar Systems Part II: Waveform Properties", IEE Proceedings of Radar Sonar Navigation, Vol. 152, No. 3, June 2005.

[14] Howland, P.F., "Target Tracking Using Television Based Bistatic Radar", IEE Proceedings of Radar Sonar Navigation, Vol. 146, No. 3, 1999

[15] Krysik, P., Samczynski, P., Malanowski, M., "Detection of Fast Maneuvering Air Targets Using GSM Based Passive Radar", International Radar Symposium 2012

[17] Mojarrabi, B., Homer, J., Kubik, K., Longstaff, I.D., "Power budget study for passive target detection and imaging using secondary applications of GPS signals in bistatic radar systems", IEEE 2002 